\title{
Enantioselective and aerobic oxidative coupling of 2-naphthol derivatives using chiral dinuclear vanadium(V) complex in water
}

\author{
Makoto Sako, Shinobu Takizawa*,Yasushi Yoshida, and Hiroaki Sasai* \\ The Institute of Scientific and Industrial Research (ISIR), Osaka University, Mihogaoka, Ibaraki, Osaka 567-0047, Japan
}

\begin{abstract}
The enantioselective oxidative coupling of 2-naphthols in water was established using dinuclear vanadium(V/IV) catalysis with $\mathrm{O}_{2}$ as the sole co-oxidant. In the vanadium-catalyzed reaction, the corresponding coupling products were obtained in good to excellent yields with up to $94 \%$ enantiomeric excess. In water, racemization of the coupling product was suppressed even at high temperature $\left(70{ }^{\circ} \mathrm{C}\right)$.
\end{abstract}

\section{Introduction}

Water is a much safer reaction solvent compared to toxic and/or flammable organic solvents, making aqueous processes environmentally benign methodologies. To date, a number of efficient, metal-catalyzed reactions and organocatalyzed reactions in water have been reported. ${ }^{1}$ The preparation of enantiomerically pure 1,1'-bi-2-naphthol (BINOL) and its derivatives are of great importance due to their wide utility in asymmetric synthesis. ${ }^{2}$ Among the synthetic methods used to access enantiomerically pure BINOLs, asymmetric and catalytic oxidative coupling of 2 naphthols is one of the most straightforward processes. ${ }^{3,4}$ We previously developed the dinuclear vanadium(V) complex $\left(R_{\mathrm{a}}, S, S\right)$-1 (Figure 1), which can catalyze the oxidative coupling of 2-naphthols through a dual activation mechanism. ${ }^{4 \mathrm{~m}-4 \mathrm{p}}\left(R_{\mathrm{a}}, S, S\right)-\mathbf{1}$ can be easily prepared in a onepot, single operation reaction from a chiral binaphthyl derivative, amino acids, and vanadium salts in $\mathrm{MeOH}$ under an $\mathrm{O}_{2}$ atmosphere and reflux conditions. The vanadium complex is stable in hydrophilic, polar solvents such as $\mathrm{MeOH}$ and $\mathrm{EtOH}$ in air. Thus we envisioned that in water, the chiral dinuclear vanadium(V) complex could promote the oxidative coupling of 2-naphthols to give BINOLs in high yields and with high enantioselectivities. To date, several procedures for the oxidative coupling of 2naphthols in water have been developed using $\mathrm{Ru}(\mathrm{OH})_{\mathrm{x}} / \mathrm{Al}_{2} \mathrm{O}_{3}{ }^{5}$, biopolymer-supported copper, ${ }^{6}$ or ferric hydrogensulfate. ${ }^{7}$ These protocols produced BINOL, but only as a racemic form. In 2015, Adão and Pessoa et al. reported the enantioselective oxidative coupling of 2 naphthol using amino acid-derived $\mathrm{Cu}(\mathrm{II})$ catalysts in EtOH/ $/ \mathrm{H}_{2} \mathrm{O}$ to provide BINOL (up to $33 \%$ yield, $44 \%$ ee). ${ }^{8}$ However, the yield and enantiomeric excess of BINOL remain unsatisfactory. Therefore, the development of an efficient oxidative coupling of 2-naphthols in water with high enantiocontrol has been a challenge. Herein, we report the highly enantioselective oxidative coupling of 2naphthols in water. Under $\mathrm{O}_{2}$ in water, the dinuclear vanadium(V) complex $\left(R_{\mathrm{a}}, S, S\right)-\mathbf{1}$ promotes oxidative coupling of 2-naphthols, to afford the corresponding coupling products in good yields with high enantioselectivities.

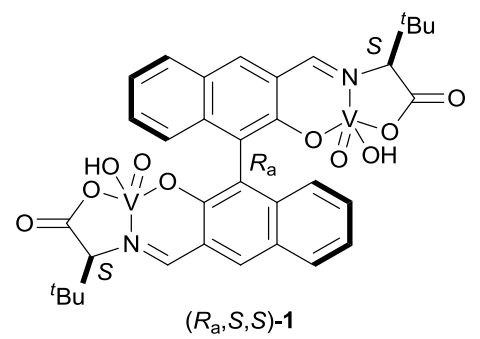

Figure 1. Dinuclear vanadium $(\mathrm{V})$ complex $\left(R_{\mathrm{a}}, S, S\right)-\mathbf{1}$

\section{Results and discussion}

Initially, the optimization of the reaction conditions for the oxidative coupling of 2-naphthol $\mathbf{2 a}$ was performed with $\left(R_{\mathrm{a}}, S, S\right)-\mathbf{1}(5 \mathrm{~mol} \%)$ in water (Table 1$)$. We previously reported that the oxidative coupling of $\mathbf{2} \mathbf{a}$ in the presence of $5 \mathrm{~mol} \%\left(R_{\mathrm{a}}, S, S\right)-\mathbf{1}$ in $\mathrm{CH}_{2} \mathrm{Cl}_{2}$ under air at $30{ }^{\circ} \mathrm{C}$ afforded $(S)$-BINOL 3a in quantitative yield with $90 \%$ ee (entry 9). ${ }^{4 \mathrm{n}}$ In contrast, either no reaction or low conversion was observed in aqueous media, most likely due to the low solubility of 2-naphthol in water (entries 1 and 2). To improve the solubility, a catalytic amount of surfactant, such as anionic surfactant sodium dodecyl sulfate (SDS), cationic surfactant tetrabutylammonium bromide (TBAB), or neutral surfactant polyoxyethylene $p$ - $t$-octylphenyl ether (Triton X-100) was added (entries 3-5), although almost no improvement in either the chemical yield or enantioselectivity was observed. However, when the

\footnotetext{
* Corresponding author. Tel.: +81-6-6879-8466; fax: +81-6-6879-8469; e-mail: taki@ sanken.osaka-u.ac.jp
} 
temperature was raised to $50{ }^{\circ} \mathrm{C}, \mathbf{3 a}$ was isolated in $91 \%$ yield with $80 \%$ ee (entry 6). When the temperature was increased to over $90{ }^{\circ} \mathrm{C}$, the product's ee drastically decreased due to decomposition of the complex (entry 7). Without $\left(R_{\mathrm{a}}, S, S\right)-\mathbf{1}$, no reaction was observed under the optimized reaction conditions (entry 8). Based on these results, efficient dual activation of 2-naphthol using $\left(R_{\mathrm{a}}, S, S\right)-1$ in water requires a temperature of at least $50{ }^{\circ} \mathrm{C}$ to promote a single electron transfer from 2-naphthol to the catalyst. $^{4 \mathrm{~m}-4 \mathrm{p}}$

Table 1 Optimization of reaction conditions for the oxidative coupling of 2-naphthol $\mathbf{2 a}$ in water
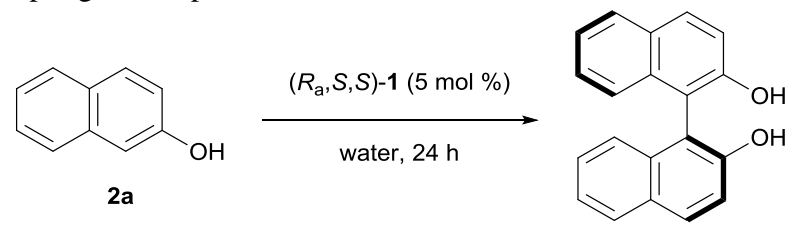

(S)-3a

\begin{tabular}{llllll}
\hline Entry & $\begin{array}{l}\text { Air or } \\
\mathrm{O}_{2}\end{array}$ & $\begin{array}{l}\text { Temp. } \\
\left({ }^{\circ} \mathrm{C}\right)\end{array}$ & $\begin{array}{l}\text { Additive } \\
(5 \mathrm{~mol} \%)\end{array}$ & $\%$ yield $^{a}$ & \% ee $^{b}$ \\
\hline 1 & Air & 30 & None & $\mathrm{NR}$ & - \\
2 & $\mathrm{O}_{2}$ & 30 & None & 11 & Rac. \\
3 & $\mathrm{O}_{2}$ & 30 & SDS & 9 & Rac. \\
4 & $\mathrm{O}_{2}$ & 30 & TBAB & 5 & 17 \\
5 & $\mathrm{O}_{2}$ & 30 & Triton X-100 & 26 & 25 \\
6 & $\mathrm{O}_{2}$ & 50 & None & $92(91)^{c}$ & 80 \\
7 & $\mathrm{O}_{2}$ & 90 & None & $>99$ & 66 \\
$8^{d}$ & $\mathrm{O}_{2}$ & 50 & None & NR & - \\
$9^{e}$ & Air & 30 & None & quant & 90 \\
\hline
\end{tabular}

${ }^{a 1} \mathrm{H}$ NMR yield, 1,3,5-trimethoxybenzene was used as an internal standard.

${ }^{b}$ Determined by HPLC.

${ }^{c}$ Yield of isolated product.

${ }^{d}$ Without $\left(R_{\mathrm{a}}, S, S\right)-\mathbf{1}$.

${ }^{e}$ Result when the reaction was carried out in $\mathrm{CH}_{2} \mathrm{Cl}_{2}$. ${ }^{4 \mathrm{n}}$

Table 2 Oxidative coupling of 2-naphthol derivatives catalyzed by dinuclear vanadium complex in water
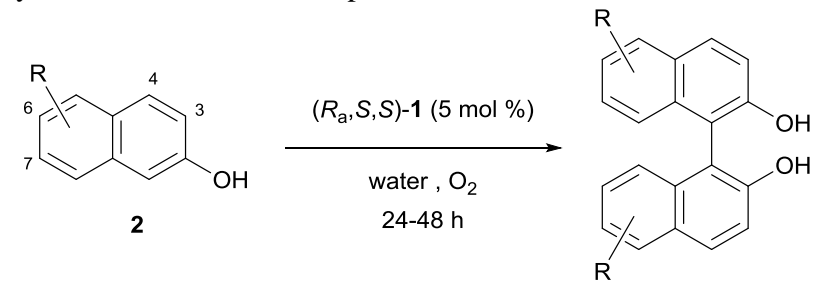

3

\begin{tabular}{lllll}
\hline Entry & Substrate, R & $\begin{array}{l}\text { Temp. } \\
\left({ }^{\circ} \mathrm{C}\right)\end{array}$ & $\begin{array}{l}\text { Yield } \\
(\%)^{a}\end{array}$ & $\begin{array}{l}\text { Ee } \\
(\%)^{b}\end{array}$ \\
\hline 1 & 2a, H & 50 & $\mathbf{3 a}, 91$ & $80(S)$ \\
2 & 2b, 7-OMOM & 50 & $\mathbf{3 b}, 78$ & $83(S)$ \\
3 & 2c, 7-OMEM & 50 & $\mathbf{3 c}, 65$ & $63(S)$ \\
4 & 2d, 7-OCH $\mathrm{CHCH}_{2}$ & 50 & $\mathbf{3 d}, 78$ & $85(S)$ \\
5 & 2e, 7-Br & 70 & $\mathbf{3 e}, 85$ & $73(S)$ \\
6 & 2f, 7-OMe & 50 & $\mathbf{3 f}, 87$ & $94(S)$ \\
7 & 2g, 6-OMe & 70 & $\mathbf{3 g}, 95$ & $63(S)$ \\
8 & 2h, 6-Me & 70 & $\mathbf{3 h}, 89$ & $77(S)$ \\
9 & 2i, 4-Ph & 70 & $\mathbf{3 i}, 82$ & $85(R)$ \\
10 & 2j, 3-OMe & 70 & $\mathbf{3 j ,} 69$ & $44(S)$ \\
\hline
\end{tabular}

${ }^{a}$ Isolated product yield. ${ }^{b}$ Determined by HPLC.
To elucidate the applicability of the oxidative coupling using the vanadium complex in water, various 2-naphthol derivatives were examined (Table 2). 2-Naphthols bearing electron-donating or withdrawing groups such as MOM-O, MEM-O, $\mathrm{CH}_{2}=\mathrm{CHCH}_{2} \mathrm{O}, \mathrm{Br}, \mathrm{MeO}, \mathrm{Me}$, or $\mathrm{Ph}$, at the $\mathrm{C} 7$, $\mathrm{C} 6$, or $\mathrm{C} 4$ position underwent the coupling reaction to produce the corresponding BINOL derivatives $\mathbf{3 b}-\mathbf{3 i}$ in good yields with high enantioselectivities (entries 2-9). 3MeO-2-naphthol $\mathbf{2 j}$ was also converted into coupling product $3 \mathbf{j}$ in good yield with moderate enantioselectivity (entry 10). The reactions of $\mathbf{2 e}$ and $\mathbf{2 g}-\mathbf{j}$ had to be carried out at higher temperature $\left(70{ }^{\circ} \mathrm{C}\right)$ and longer reaction time (48 h) due to the low solubility of $\mathbf{2 e}$ and $\mathbf{2 g}$-j in water.

In an effort to clarify whether racemization of the products occurs, optically active 7,7'-dimethoxy-substituted BINOL 3f $[94 \%$ ee $(S)]$ or 6,6'-dimethoxy-substituted BINOL 3g [81\% ee $(S)]$ was stirred in water at $70{ }^{\circ} \mathrm{C}$ under the coupling conditions [100 mol \% 2-naphthol 2a and 5 mol \% dinuclear vanadium(V) complex $\left.\left(R_{\mathrm{a}}, S, S\right)-\mathbf{1}\right]$. After $24 \mathrm{~h}$, the dimethoxy-substituted BINOLs were recovered with $93 \%$ ee for $(S)$-3f and $77 \%$ ee for $(S)-\mathbf{3 g} .{ }^{9}$ Under the optimal reaction conditions in water, the ee of $\mathbf{3 f}$ and $\mathbf{3 g}$ decreased slightly. However, using $\mathrm{ClCH}_{2} \mathrm{CH}_{2} \mathrm{Cl}$ as the reaction solvent instead of water resulted in decreasing ee; 3f: from $94 \%$ ee $(S)$ to $74 \%$ ee $(S)$; $3 \mathbf{g}$ : from $81 \%$ ee $(S)$ to $11 \%$ ee $(R) .{ }^{10}$ It should be noted that in water, racemization of BINOLs is significantly suppressed as compared to an organic solvent. $^{11}$

To prove the utility of the present methodology, the oxidative coupling of $\mathbf{2}$ on a gram scale was examined (Scheme 1). The reaction using $1.2 \mathrm{~g}$ of $\mathbf{2 a}$ or $\mathbf{2 f}$ in the presence of $5 \mathrm{~mol} \%\left(R_{\mathrm{a}}, S, S\right)-\mathbf{1}$ allowed the formation of $(S)-3 a$ in $83 \%$ yield with $77 \%$ ee and $(S)-3 f$ in $63 \%$ yield with $94 \%$ ee, respectively (eqs. 1 and 2).

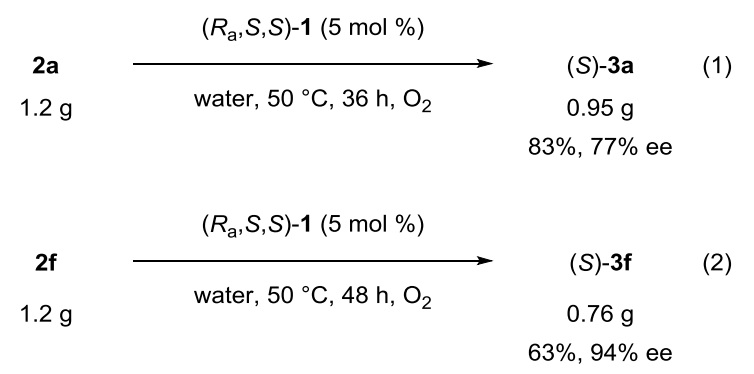

Scheme 1 Oxidative coupling of 2-naphthols catalyzed by dinuclear vanadium complex in water on a gram scale

\section{Conclusion}

In conclusion, we have developed the first example of a highly efficient and enantioselective oxidative coupling of 2-naphthols 2 in water catalyzed by a chiral dinuclear vanadium(V) complex. In water, $\left(R_{\mathrm{a}}, S, S\right)$-1 maintained high catalytic activity for the coupling reaction to produce BINOLs 3 in high yields with up to $94 \%$ ee. In water, almost no racemization of BINOLs was observed at $70{ }^{\circ} \mathrm{C}$. Furthermore, a gram scale synthesis of BINOLs in aqueous 
media was achieved with good yields and high enantioselectivities.

\section{Experimental}

\subsection{General}

${ }^{1} \mathrm{H}$ - and ${ }^{13} \mathrm{C}-\mathrm{NMR}$ and spectra were recorded with JEOL JMN ECS400 FT NMR, JNM ECA600 FT NMR or Bruker AVANCE II $\left({ }^{1} \mathrm{H}-\mathrm{NMR} 400,600\right.$ or $700 \mathrm{MHz},{ }^{13} \mathrm{C}-\mathrm{NMR}$ 100, 150 or $175 \mathrm{MHz}$ ). ${ }^{1} \mathrm{H}-\mathrm{NMR}$ spectra are reported as follows: chemical shift in ppm relative to the chemical shift of TMS at $0 \mathrm{ppm}$, integration, multiplicities ( $\mathrm{s}=$ singlet, $\mathrm{d}$ $=$ doublet, $\mathrm{t}=$ triplet, $\mathrm{m}=$ multiplet ), and coupling constants $(\mathrm{Hz}) .{ }^{13} \mathrm{C}-\mathrm{NMR}$ spectra are reported in ppm relative to the central line of triplet for $\mathrm{CDCl}_{3}$ at $77 \mathrm{ppm}$. Optical rotations were measured with JASCO P-1030 polarimeter. HPLC analyses were performed on a JASCO HPLC system (JASCO PU 980 pump and UV-975 UV/Vis detector) using a mixture of hexane and 2-propanol as eluents. Column chromatography on $\mathrm{SiO}_{2}$ was performed with Kishida Silica Gel $(63-200 \mu \mathrm{m})$. Commercially available organic and inorganic compounds were used without further purification. $\left(R_{\mathrm{a}}, S, S\right)-\mathbf{1}, \mathbf{2 b}, \mathbf{2 d}, \mathbf{2} \mathbf{h}, \mathbf{2} \mathbf{i}$ and $\mathbf{2} \mathbf{j}$ were prepared following the reported procedures. ${ }^{40,12}$ Products $\mathbf{3 a}, \mathbf{3 b}, \mathbf{3 d}$, 3e, 3f, 3g, 3h, 3i and $\mathbf{3 j}$ were identical in all respects with the data reported in the literature. ${ }^{4 n, 13}$ Absolute configurations were assigned by comparison of the specific rotation reported in the literature. ${ }^{4 n, 13}$

\subsection{Preparation of 7-((2-methoxyethoxy)methoxy)-2- naphthol 2c}

To a solution of 2,7-dihydroxynaphthalene (961 mg, 6.0 $\mathrm{mmol})$ in $\mathrm{CH}_{2} \mathrm{Cl}_{2}(30 \mathrm{~mL})$ were added ${ }^{i} \mathrm{Pr}_{2} \mathrm{NEt}(1.15 \mathrm{~mL}$, $6.6 \mathrm{mmol})$ and $\mathrm{MEMCl}(0.75 \mathrm{~mL}, 6.6 \mathrm{mmol})$ at $0{ }^{\circ} \mathrm{C}$. The mixture was warmed to $\mathrm{rt}$ and then stirred for $24 \mathrm{~h}$. After the reaction was completed, water was added to the reaction mixture and the solution was extracted with $\mathrm{CH}_{2} \mathrm{Cl}_{2}$. The organic layer was washed with brine, dried over $\mathrm{Na}_{2} \mathrm{SO}_{4}$, and evaporated in vacuo. After the purification via $\mathrm{SiO}_{2}$ column chromatography (hexane/acetone $=3 / 1$ ), the desired product was obtained as a yellow oil $(675 \mathrm{mg}, 45 \%) .{ }^{1} \mathrm{H}$ NMR $\left(400 \mathrm{MHz}, \mathrm{CDCl}_{3}\right): \delta$ $7.67(\mathrm{~d}, J=8.7,2 \mathrm{H}), 7.27(\mathrm{~d}, J=2.3 \mathrm{~Hz}, 1 \mathrm{H}), 7.05(\mathrm{dd}, J=$ 8.7, $2.3 \mathrm{~Hz}, 1 \mathrm{H}), 7.04(\mathrm{~d}, J=2.3 \mathrm{~Hz}, 1 \mathrm{H}), 6.96(\mathrm{dd}, J=8.7$, $2.3 \mathrm{~Hz}, 1 \mathrm{H}), 5.37(\mathrm{~s}, 2 \mathrm{H}), 5.01(\mathrm{~s}, 1 \mathrm{H}), 3.88-3.86(\mathrm{~m}, 2 \mathrm{H})$, 3.60-3.57 (m, 2H), $3.39(\mathrm{~s}, 3 \mathrm{H}) ;{ }^{13} \mathrm{C}$ NMR $(100 \mathrm{MHz}$, $\left.\mathrm{CDCl}_{3}\right): \delta 155.2(\mathrm{C}), 154.4(\mathrm{C}), 135.6(\mathrm{C}), 129.1(\mathrm{CH})$, $129.1(\mathrm{CH}), 124.4(\mathrm{C}), 116.0(\mathrm{CH}), 115.9(\mathrm{CH}), 108.7$ $(\mathrm{CH}), 108.4(\mathrm{CH}), 93.1\left(\mathrm{CH}_{2}\right), 71.3\left(\mathrm{CH}_{2}\right), 67.3\left(\mathrm{CH}_{2}\right), 58.6$ $\left(\mathrm{CH}_{3}\right)$; HRMS (ESI) calcd for $\mathrm{C}_{14} \mathrm{H}_{16} \mathrm{NaO}_{4}, \mathrm{~m} / z=271.0946$ $\left[(\mathrm{M}+\mathrm{Na})^{+}\right]$, found $\mathrm{m} / z=271.0938 ; \mathrm{IR}(\mathrm{KBr}): v 3360,3062$, $2931,1635,1515,1448,1200,1159,1007,833 \mathrm{~cm}^{-1}$.

\subsection{General procedure for coupling reactions of 2- naphthols 2 using $\left(R_{\mathrm{a}}, S, S\right)-1$ in water}

A test tube was charged with a water $(1 \mathrm{~mL})$ heterogeneous solution of coupling substrate $2(0.2 \mathrm{mmol})$ under $\mathrm{O}_{2}(1 \mathrm{~atm})$ atmosphere. Vanadium catalyst $\left(R_{\mathrm{a}}, S, S\right)-\mathbf{1}$ (0.01 mmol, $5 \mathrm{~mol} \%$ ) was added to the solution. The reaction mixture was stirred at $50{ }^{\circ} \mathrm{C}$ for $\mathbf{2 a}, \mathbf{2 b}, \mathbf{2 c}, \mathbf{2 d}$ and $\mathbf{2 f}$ or $70{ }^{\circ} \mathrm{C}$ for $\mathbf{2 e}, \mathbf{2 g}, \mathbf{2 h}, \mathbf{2 i}$ and $\mathbf{2 j}$ until the reaction had reached completion by monitoring with TLC analysis. The reaction mixture was then directly purified by silica gel column chromatography eluting with ethyl acetate/hexane to give the coupling product.

\section{4. (S)-1,1'-Bi-2-naphthol $3 \mathbf{a}^{4 \mathrm{n}}$}

Reaction time: $24 \mathrm{~h}$; Reaction temperature: $50{ }^{\circ} \mathrm{C}$; $91 \%$ yield; $[\alpha]_{\mathrm{D}}{ }^{22}=-26.9$ (c 1.0, THF, 80\% ee); ${ }^{1} \mathrm{H}$ NMR (400 $\left.\mathrm{MHz}, \mathrm{CDCl}_{3}\right): \delta 7.98(\mathrm{~d}, J=8.7 \mathrm{~Hz}, 2 \mathrm{H}), 7.89(\mathrm{~d}, J=7.8$ $\mathrm{Hz}, 2 \mathrm{H}), 7.43-7.34(\mathrm{~m}, 4 \mathrm{H}), 7.31(\mathrm{td}, J=7.8 \mathrm{~Hz}, 1.6 \mathrm{~Hz}$, 2H), $7.15(\mathrm{~d}, J=8.2 \mathrm{~Hz}, 2 \mathrm{H}), 5.04(\mathrm{~s}, 2 \mathrm{H})$; The enantiometric excess was determined by HPLC with a Daicel Chiralpak AS-H column (hexane:2-propanol = 7:1, $\lambda=229 \mathrm{~nm}$, flow rate $=1.0 \mathrm{~mL} / \mathrm{min}) ; t_{R}$ (major enantiomer) $=9.8 \mathrm{~min}, t_{R}($ minor enantiomer $)=15.1 \mathrm{~min}, 80 \%$ ee.

\section{5. (S)-7,7'-Bis(methoxymethoxy)-1,1'-bi-2-naphthol $3 \mathbf{b}^{4 \mathrm{n}}$}

Reaction time: $48 \mathrm{~h}$; Reaction temperature: $50{ }^{\circ} \mathrm{C} ; 78 \%$ yield; $[\alpha]_{\mathrm{D}}{ }^{13}=+127.6$ (c $1.1, \mathrm{CHCl}_{3}, 83 \%$ ee); ${ }^{1} \mathrm{H} \mathrm{NMR}$ $\left(400 \mathrm{MHz}, \mathrm{CDCl}_{3}\right): \delta 7.87(\mathrm{~d}, J=8.7 \mathrm{~Hz}, 2 \mathrm{H}), 7.79(\mathrm{~d}, J=$ $8.7 \mathrm{~Hz}, 2 \mathrm{H}), 7.21(\mathrm{~d}, J=8.7 \mathrm{~Hz}, 2 \mathrm{H}), 7.14(\mathrm{dd}, J=8.7,2.3$ $\mathrm{Hz}, 2 \mathrm{H}), 6.65$ (d, $J=2.3 \mathrm{~Hz}, 2 \mathrm{H}), 5.08$ (s, 2H), 4.99 (s, 4H), $3.31(\mathrm{~s}, 6 \mathrm{H})$; The enantiometric excess was determined by HPLC with a Daicel Chiralpak AS-H column (hexane:2propanol $=7: 1, \lambda=235 \mathrm{~nm}$, flow rate $=1.0 \mathrm{~mL} / \mathrm{min}) ; t_{R}$ $($ major enantiomer $)=16.5 \mathrm{~min}, t_{R}($ minor enantiomer $)=$ $27.9 \mathrm{~min}, 83 \%$ ee.

\section{6. (S)-7,7'-Bis((2-methoxyethoxy)methoxy)-1,1'-bi-2- naphthol 3c}

Reaction time: $48 \mathrm{~h}$; Reaction temperature: $50{ }^{\circ} \mathrm{C}$; $65 \%$ yield, yellow oil; $[\alpha]_{\mathrm{D}}{ }^{22}=+61.9\left(c 0.7, \mathrm{CHCl}_{3}, 63 \%\right.$ ee $) ;{ }^{1} \mathrm{H}$ NMR (400 MHz, $\left.\mathrm{CDCl}_{3}\right): \delta 7.88(\mathrm{~d}, J=9.2 \mathrm{~Hz}, 2 \mathrm{H}), 7.80$ $(\mathrm{d}, J=8.7 \mathrm{~Hz}, 2 \mathrm{H}), 7.26(\mathrm{~s}, 2 \mathrm{H}), 7.23(\mathrm{~d}, J=9.2 \mathrm{~Hz}, 2 \mathrm{H})$, $7.16(\mathrm{dd}, J=8.7,2.3 \mathrm{~Hz}, 2 \mathrm{H}), 6.71(\mathrm{~d}, J=2.3 \mathrm{~Hz}, 2 \mathrm{H})$, $5.15(\mathrm{~s}, 2 \mathrm{H}), 5.10(\mathrm{~s}, 4 \mathrm{H}), 3.70-3.62(\mathrm{~m}, 4 \mathrm{H}), 3.45-3.34(\mathrm{~m}$, $4 \mathrm{H}), 3.28(\mathrm{~s}, 6 \mathrm{H}) ;{ }^{13} \mathrm{C}$ NMR $\left(100 \mathrm{MHz}, \mathrm{CDCl}_{3}\right): \delta 156.4$ (C), $153.3(\mathrm{C}), 134.6(\mathrm{C}), 131.1(\mathrm{CH}), 130.0(\mathrm{CH}), 125.4$ (C), $116.0(\mathrm{CH}), 115.8(\mathrm{CH}), 110.2(\mathrm{C}), 107.9(\mathrm{CH}), 93.4$ $\left(\mathrm{CH}_{2}\right), 71.4\left(\mathrm{CH}_{2}\right), 67.5\left(\mathrm{CH}_{2}\right), 59.0\left(\mathrm{CH}_{3}\right)$; HRMS (ESI) calcd for $\left.\mathrm{C}_{28} \mathrm{H}_{30} \mathrm{NaO}_{8}, m / z=517.1838(\mathrm{M}+\mathrm{Na})^{+}\right]$, found $m / z=517.1826$; IR (KBr): v 3390, 3058, 2931, 1621, 1512, 1203, 1159, 1019, 982, $834 \mathrm{~cm}^{-1}$; The enantiometric excess was determined by HPLC with a Daicel Chiralpak AS-H column (hexane:2-propanol $=4: 1, \lambda=220 \mathrm{~nm}$, flow rate $=$ $1.0 \mathrm{~mL} / \mathrm{min}) ; t_{R}$ (major enantiomer) $=22.0 \mathrm{~min}, t_{R}$ (minor enantiomer $)=30.1 \mathrm{~min}, 63 \%$ ee.

\section{7. (S)-7,7'-Bis(allyloxy)-1,1'-bi-2-naphthol $3 d^{4 \mathrm{n}}$}

Reaction time: $48 \mathrm{~h}$; Reaction temperature: $50{ }^{\circ} \mathrm{C} ; 78 \%$ yield; $[\alpha]_{\mathrm{D}}{ }^{22}=+177.6$ (c $1.5, \mathrm{CHCl}_{3}, 85 \%$ ee); ${ }^{1} \mathrm{H} \mathrm{NMR}$ $\left(400 \mathrm{MHz}, \mathrm{CDCl}_{3}\right): \delta 7.85(\mathrm{~d}, J=8.7 \mathrm{~Hz}, 2 \mathrm{H}), 7.77(\mathrm{~d}, J=$ $8.7 \mathrm{~Hz}, 2 \mathrm{H}), 7.20(\mathrm{~d}, J=8.7 \mathrm{~Hz}, 2 \mathrm{H}), 7.04(\mathrm{dd}, J=8.7,2.4$ 
$\mathrm{Hz}, 2 \mathrm{H}), 6.47$ (d, $J=2.4 \mathrm{~Hz}, 2 \mathrm{H}), 5.90-5.80(\mathrm{~m}, 2 \mathrm{H}), 5.15-$ $5.09(\mathrm{~m}, 4 \mathrm{H}), 5.06(\mathrm{~s}, 2 \mathrm{H}), 4.32-4.21(\mathrm{~m}, 4 \mathrm{H})$; The enantiometric excess was determined by HPLC with a Daicel Chiralpak IA column (hexane:2-propanol $=7: 1, \lambda=$ $235 \mathrm{~nm}$, flow rate $=1.0 \mathrm{~mL} / \mathrm{min}) ; t_{R}$ (major enantiomer $)=$ $12.2 \mathrm{~min}, t_{R}$ (minor enantiomer $)=22.7 \mathrm{~min}, 85 \%$ ee.

\section{8. (S)-7,7'-Dibromo-1,1'-bi-2-naphthol 3e $\mathrm{e}^{13 \mathrm{a}}$}

Reaction time: $48 \mathrm{~h}$; Reaction temperature: $70{ }^{\circ} \mathrm{C} ; 85 \%$ yield; $[\alpha]_{\mathrm{D}}{ }^{21}=+129.1$ (c 1.8, $\mathrm{CHCl}_{3}, 73 \%$ ee); ${ }^{1} \mathrm{H}$ NMR $\left(400 \mathrm{MHz}, \mathrm{CDCl}_{3}\right): \delta 7.94(\mathrm{~d}, J=9.2 \mathrm{~Hz}, 2 \mathrm{H}), 7.76(\mathrm{~d}, J=$ $8.7 \mathrm{~Hz}, 2 \mathrm{H}), 7.47(\mathrm{dd}, J=8.7,1.8 \mathrm{~Hz}, 2 \mathrm{H}), 7.38(\mathrm{~d}, J=9.2$ $\mathrm{Hz}, 2 \mathrm{H}), 7.23(\mathrm{~d}, J=1.8 \mathrm{~Hz}, 2 \mathrm{H}), 5.05$ (s, 2H); The enantiometric excess was determined by HPLC with a Daicel Chiralcel OD-H column (hexane:2-propanol $=9: 1, \lambda$ $=235 \mathrm{~nm}$, flow rate $=1.0 \mathrm{~mL} / \mathrm{min}) ; t_{R}$ (major enantiomer) $=15.9 \mathrm{~min}, t_{R}($ minor enantiomer $)=33.4 \mathrm{~min}, 73 \% \mathrm{ee}$.

\section{9. (S)-7,7'-Dimethoxy-1,1'-bi-2-naphthol $3 \mathrm{f}^{4 \mathrm{n}}$}

Reaction time: $48 \mathrm{~h}$; Reaction temperature: $50{ }^{\circ} \mathrm{C} ; 87 \%$ yield; $[\alpha]_{\mathrm{D}}{ }^{22}=+122.3\left(\right.$ c $1.0, \mathrm{CHCl}_{3}, 94 \%$ ee $) ;{ }^{1} \mathrm{H}$ NMR $\left(400 \mathrm{MHz}, \mathrm{CDCl}_{3}\right): \delta 7.86(\mathrm{~d}, J=8.7 \mathrm{~Hz}, 2 \mathrm{H}), 7.77(\mathrm{~d}, J=$ $8.7 \mathrm{~Hz}, 2 \mathrm{H}), 7.21(\mathrm{~d}, J=8.7 \mathrm{~Hz}, 2 \mathrm{H}), 7.02(\mathrm{dd}, J=8.7,2.7$ $\mathrm{Hz}, 2 \mathrm{H}), 6.47(\mathrm{~d}, J=2.7 \mathrm{~Hz}, 2 \mathrm{H}), 5.04(\mathrm{~s}, 2 \mathrm{H}), 3.56(\mathrm{~s}$, $6 \mathrm{H})$; The enantiometric excess was determined by HPLC with a Daicel Chiralpak AS-H column (hexane:2-propanol $=9: 1, \lambda=235 \mathrm{~nm}$, flow rate $=1.0 \mathrm{~mL} / \mathrm{min}) ; t_{R}$ (major enantiomer $)=18.8 \mathrm{~min}, t_{R}($ minor enantiomer $)=28.0 \mathrm{~min}$, $94 \%$ ee.

\subsection{0. (S)-6,6'-Dimethoxy-1,1'-bi-2-naphthol $3 \mathrm{~g}^{4 \mathrm{n}}$}

Reaction time: $48 \mathrm{~h}$; Reaction temperature: $70{ }^{\circ} \mathrm{C}$; $95 \%$ yield; $[\alpha]_{\mathrm{D}}{ }^{20}=+25.6\left(c 1.6, \mathrm{CHCl}_{3}, 63 \%\right.$ ee $) ;{ }^{1} \mathrm{H}$ NMR $(400$ $\left.\mathrm{MHz} \mathrm{CDCl}_{3}\right): \delta 7.83(\mathrm{dd}, J=9.2,1.8 \mathrm{~Hz}, 2 \mathrm{H}), 7.33(\mathrm{dd}, J$ $=9.2,1.8 \mathrm{~Hz}, 2 \mathrm{H}), 7.19(\mathrm{~s}, 2 \mathrm{H}), 7.05(\mathrm{~d}, J=9.2 \mathrm{~Hz}, 2 \mathrm{H})$, $6.97(\mathrm{dd}, J=9.2,1.8 \mathrm{~Hz}, 2 \mathrm{H}), 4.94(\mathrm{~s}, 2 \mathrm{H}), 3.89$ (s, 6H); The enantiometric excess was determined by HPLC with a Daicel Chiralpak AS column (hexane:2-propanol $=4: 1, \lambda=$ $260 \mathrm{~nm}$, flow rate $=1.0 \mathrm{~mL} / \mathrm{min}) ; t_{R}$ (major enantiomer $)=$ $12.1 \mathrm{~min}, t_{R}($ minor enantiomer $)=21.8 \mathrm{~min}, 63 \% \mathrm{ee}$.

\subsection{1. (S)-6,6'-Dimethyl-1,1'-bi-2-naphthol $3 h^{4 n}$}

Reaction time: $48 \mathrm{~h}$; Reaction temperature: $70{ }^{\circ} \mathrm{C}$; $89 \%$ yield; $[\alpha]_{\mathrm{D}}{ }^{17}=+51.9\left(c 0.7, \mathrm{CHCl}_{3}, 77 \%\right.$ ee $) ;{ }^{1} \mathrm{H}$ NMR $(400$ $\left.\mathrm{MHz}, \mathrm{CDCl}_{3}\right): \delta 7.88(\mathrm{~d}, J=9.2 \mathrm{~Hz}, 2 \mathrm{H}), 7.66(\mathrm{~s}, 2 \mathrm{H}), 7.34$ $(\mathrm{d}, J=9.2 \mathrm{~Hz}, 2 \mathrm{H}), 7.14(\mathrm{~d}, J=8.2 \mathrm{~Hz}, 2 \mathrm{H}), 7.05$ (d, $J=$ $8.2 \mathrm{~Hz}, 2 \mathrm{H}), 4.96$ (s, 2H), 2.47 (s, 6H); The enantiometric excess was determined by HPLC with a Daicel Chiralpak AS-H column (hexane:2-propanol $=7: 1, \lambda=229 \mathrm{~nm}$, flow rate $=1.0 \mathrm{~mL} / \mathrm{min}) ; t_{R}$ (major enantiomer) $=8.2 \mathrm{~min}, t_{R}$ $($ minor enantiomer $)=12.9 \mathrm{~min}, 77 \%$ ee.

\subsection{2. (R)-4,4'-Diphenyl-1,1'-bi-2-naphthol $3 i^{13 b}$}

Reaction time: $48 \mathrm{~h}$; Reaction temperature: $70{ }^{\circ} \mathrm{C} ; 82 \%$ yield; $[\alpha]_{\mathrm{D}}{ }^{22}=-22.1$ (c 1.0, $\mathrm{CHCl}_{3}, 85 \%$ ee $) ;{ }^{1} \mathrm{H}$ NMR $(400$ $\left.\mathrm{MHz}, \mathrm{CDCl}_{3}\right): \delta$ 7.97-7.94 (m, 2H), 7.64-7.48 (m, 10H), $7.38(\mathrm{~s}, 2 \mathrm{H}), 7.36-7.31(\mathrm{~m}, 6 \mathrm{H}), 5.16(\mathrm{~s}, 2 \mathrm{H})$; The enantiometric excess was determined by HPLC with a
Daicel Chiralcel OD-H column (hexane:2-propanol $=4: 1, \lambda$ $=220 \mathrm{~nm}$, flow rate $=1.0 \mathrm{~mL} / \mathrm{min}) ; t_{R}$ (minor enantiomer) $=8.4 \mathrm{~min}, t_{R}$ (major enantiomer) $=13.3 \mathrm{~min}, 85 \%$ ee.

\subsection{3. (S)-3,3'-Dimethoxy-1,1'-bi-2-naphthol $3 \mathbf{j}^{4 \mathrm{n}}$}

Reaction time: $48 \mathrm{~h}$; Reaction temperature: $70{ }^{\circ} \mathrm{C} ; 69 \%$ yield; $[\alpha]_{\mathrm{D}}{ }^{23}=-6.2\left(c 1.2, \mathrm{CHCl}_{3}, 44 \%\right.$ ee $) ;{ }^{1} \mathrm{H}$ NMR $(400$ $\left.\mathrm{MHz}, \mathrm{CDCl}_{3}\right): \delta 7.78(\mathrm{~d}, J=8.2 \mathrm{~Hz}, 2 \mathrm{H}), 7.34-7.29(\mathrm{~m}$, $4 \mathrm{H}), 7.18-7.12(\mathrm{~m}, 4 \mathrm{H}), 5.88(\mathrm{~s}, 2 \mathrm{H}), 4.09$ (s, 6H); The enantiometric excess was determined by HPLC with a Daicel Chiralpak AS column (hexane:2-propanol $=1: 1, \lambda=$ $236 \mathrm{~nm}$, flow rate $=1.0 \mathrm{~mL} / \mathrm{min}) ; t_{R}$ (major enantiomer $)=$ $19.2 \mathrm{~min}, t_{R}($ minor enantiomer $)=46.4 \mathrm{~min}, 44 \%$ ee.

\section{Acknowledgements}

This work was supported by the Advanced Catalytic Transformation program for Carbon utilization (ACTC), Core Research for Evolutionary Science and Technology (CREST), a Grant-in-Aid for Scientific Research from MEXT. We would like to thank the technical staff of the ISIR Comprehensive Analysis Centre at Osaka University for their assistance.

\section{References and notes}

1. (a) Li, C.-J. Chem. Rev. 2005, 105, 3095. (b) Raj, M.; Singh, V. K. Chem. Commun. 2009, 6687. (c) Butler, R. N.; Coyne, A. G. Chem. Rev. 2010, 110, 6302. (d) Bhowmick, S.; Bhowmick, K. C. Tetrahedron: Asymmetry 2011, 22, 1945. (e) Simon, M.-O.; Li, C.-J. Chem. Soc. Rev. 2012, 41, 1415. (f) In Science of Synthesis Water in Organic Synthesis Workbench Edition (S. Kobayashi, Ed) Thieme (2014).

2. (a) Pu, L. Chem. Rev. 1998, 98, 2405. (b) Brunel, J. M. Chem. Rev. 2007, 107, PR1. (c) In Privileged Chiral Ligands and Catalysts (Zhou, Q.-L. Ed) Wiley-VCH Verlag GmbH \& Co. KGaA (2011). (d) Allen, S. E.; Walvoord, R. R.; Padilla-Salinas, R.; Kozlowski, M. C. Chem. Rev. 2013, 113, 6234 .

3. (a) Punniyamurthy, T.; Rout, L. Coord. Chem. Rev. 2008, 252, 134. (b) Takizawa, S.; Katayama, T.; Sasai, H. Chem. Commun. 2008, 4113. (c) Kozlowski, M. C.; Morgan, B. J.; Linton, E. C. Chem. Soc. Rev. 2009, 38, 3193. (d) Takizawa, S. Chem. Pharm. Bull. 2009, 57, 1179. (e) Wang, H. Chirality 2010, 22, 827. (f) O'Brien, E. M.; Morgan, B. J.; Mulrooney, C. A.; Carroll, P. J.; Kozlowski, M. C. J. Org. Chem. 2010, 75, 57. (g) Egami, H.; Matsumoto, K.; Oguma, T.; Kunisu, T.; Katsuki, T. J. Am. Chem. Soc. 2010, 132, 13633. (h) Podlesny, E. E.; Kozlowski, M. C. Org. Lett. 2012, 14, 1408. (i) Podlesny, E. E.; Kozlowski, M. C. J. Org. Chem. 2013, 78, 466 .

4. (a) Hon, S.-W.; Li, C.-H.; Kuo, J.-H.; Barhate, N. B.; Liu, Y.-H.; Wang, Y.; Chen, C.-T. Org. Lett. 2001, 3, 869. (b) Chu, C.-Y.; Hwang, D.-R.; Wang, S.-K.; Uang, B.-J. Chem. Commun. 2001, 980. (c) Barhate, N. B.; Chen, C.-T. Org. Lett. 2002, 4, 2529. (d) Luo, Z.; Liu, Q.; Gong, L.; Cui, X.; Mi, A.; Jiang, Y. Chem. Commun. 2002, 914. (e) Luo, Z.; Liu, Q.; Gong, L.; Cui, X.; Mi, A.; Jiang, Y. Angew. Chem. Int. Ed. 2002, 41, 4532. (f) Chu, C.-Y.; Uang, B.-J. Tetrahedron: Asymmetry 2003, 14, 53. (g) Somei, H.; Asano, Y.; Yoshida, T.; Takizawa, S.; Yamataka, H.; Sasai, H. 
Tetrahedron Lett. 2004, 45, 1841. (h) Tada, M.; Taniike, T.; Kantam, L. M.; Iwasawa, Y. Chem. Commun. 2004, 2542. (i) Tada, M.; Kojima, N.; Izumi, Y.; Taniike, T.; Iwasawa, Y. J. Phys. Chem. B 2005, 109, 9905. (j) Habaue, S.; Murakami, S.; Higashimura, H. J. Polym. Sci. Part A: Polym. Chem. 2005, 43, 5872. (k) Tada, M.; Iwasawa, Y. Chem. Commun. 2006, 2833. (1) Guo, Q.-X.; Wu, Z.-J.; Luo, Z.-B.; Liu, Q.-Z.; Ye, J.-L.; Luo, S.-W.; Cun, L.-F.; Gong, L.-Z. J. Am. Chem. Soc. 2007, 129, 13927. (m) Takizawa, S.; Katayama, T.; Kameyama, C.; Onitsuka, K.; Suzuki, T.; Yanagida, T.; Kawai, T.; Sasai, H. Chem. Commun. 2008, 1810. (n) Takizawa, S.; Katayama, T.; Somei, H.; Asano, Y.; Yoshida, T.; Kameyama, C.; Rajesh, D.; Onitsuka, K.; Suzuki, T.; Mikami, M.; Yamataka, H.; Jayaprakash, D.; Sasai, H. Tetrahedron 2008, 64, 3361. (o) Takizawa, S.; Rajesh, D.; Katayama, T.; Sasai, H. Synlett 2009, 1667. (p) Takizawa, S.; Kodera, J.; Yoshida, Y.; Sako, M.; Breukers, S.; Enders, D.; Sasai, H. Tetrahedron 2014, 70, 1786.

5. Reddy, K. R.; Rajgopal, K.; Kantam, M. L. Catal. Lett. 2007, 114, 36 .

6. Eshghi, H.; Bakavoli, M.; Moradi, H. Chin. Chem. Lett. 2009, 20, 663.

7. Matsushita, M.; Kamata, K.; Yamaguchi, K.; Mizuno, N. J. Am. Chem. Soc. 2005, 127, 6632.

8. Adão, P.; Barroso, S.; Carvalho, M. F. N. N.; Teixeira, C. M.; Kuznetsov, M. L.; Pessoa, J. C. Dalton Trans. 2015, 44, 1612 .

9. After the reaction in water, $(S)$-3a was obtained quantitatively with $82 \%$ ee (recovering with $\mathbf{3 f}$ ) and $78 \%$ ee (recovering with $\mathbf{3 g}$ ), respectively.

10. After the reaction in $\mathrm{ClCH}_{2} \mathrm{CH}_{2} \mathrm{Cl}$ at $70{ }^{\circ} \mathrm{C},(S)-\mathbf{3 a}$ was obtained quantitatively with $13 \%$ ee (recovering with $\mathbf{3 f}$ ) and $23 \%$ ee (recovering with $\mathbf{3 g}$ ), respectively.

11. (a) Kyba, E. B.; Koga, K.; Sousa, L. R.; Siegel, M. G.; Cram, D. J. J. Am. Chem. Soc. 1973, 95, 2692. (b) Kyba, E. P.; Gokel, G. W.; Jong, F. D.; Koga, K.; Sousa, L. R.; Siegel, M. G.; Kaplan, L.; Sogah, G. D. Y.; Cram, D. J. J. Org. Chem. 1977, 42, 4173.

12. (a) Marsilje, T. H.; Milkiewicz, K. L.; Hangauer, D. G. Bioorg. Med. Chem. Lett. 2000, 10, 477. (b) Sugimura, T.; Matsushita, N.; Minokami, K.; Kurita, S. Tetrahedron 2007, 63, 1762. (c) Verga, D.; Percivalle, C.; Doria, F.; Porta, A.; Freccero, M. J. Org. Chem. 2011, 76, 2319. (d) Zhang, J.; Liu, Q.; Liu, X.; Zhang, S.; Jiang, P.; Wang, Y.; Luo, S.; Li, Y.; Wang, Q. Chem. Commun. 2015, 51, 1297. (e) Sivapackiam, J.; Harpstrite, S. E.; Prior, J. L.; Gu, H.; Rath, N. P.; Sharma, V. Dalton Trans. 2010, 39, 5842.

13. (a) Lustenberger, P.; Diederich, F. Helve. Chim. Acta 2000 , 83, 2865. (b) Okuma, K.; Itoyama, R.; Sou, A.; Nagahora. N.; Shioj, K. Chem. Commun. 2012, 48, 11145. 


\section{Stereochemistry Abstract}

To create your abstract, type over the instructions in the template box below.

Fonts or abstract dimensions should not be changed or altered. You may insert more abstracts by copying this box or by using the menu option to insert a stereochemistry abstract.

Makoto Sako, Shinobu Takizawa*, Yasushi Yoshida, and Hiroaki Sasai*

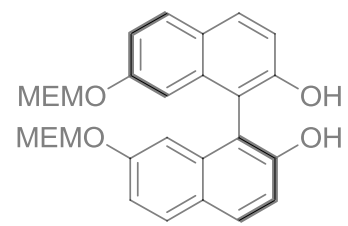

$\mathrm{Ee}=63 \%$ by HPLC on Daicel Chiralpak AS-H column $[\alpha]_{\mathrm{D}}^{22}=+61.9\left(c 0.7, \mathrm{CHCl}_{3}\right)$

Source of chirality: Enantioselective oxidative coupling Absolute configuration: $(S)$

$$
\mathrm{C}_{28} \mathrm{H}_{30} \mathrm{O}_{8}
$$

(S)-7,7'-Bis((2-methoxyethoxy)methoxy)-1,1'-bi-2-naphthol 


\section{Graphical Abstract}

To create your abstract, type over the instructions in the template box below.

Fonts or abstract dimensions should not be changed or altered.

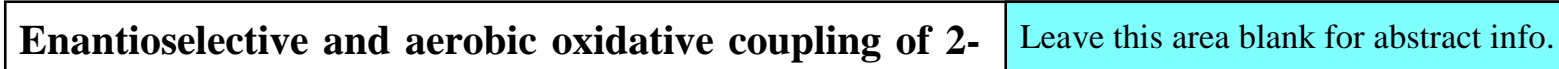
naphthol derivatives using chiral dinuclear vanadium $(V)$ complex in water

Makoto Sako, Shinobu Takizawa*, Yasushi Yoshida, and Hiroaki Sasai*

The Institute of Scientific and Industrial Research (ISIR), Osaka University, Mihogaoka, Ibaraki, Osaka 567-0047, Japan

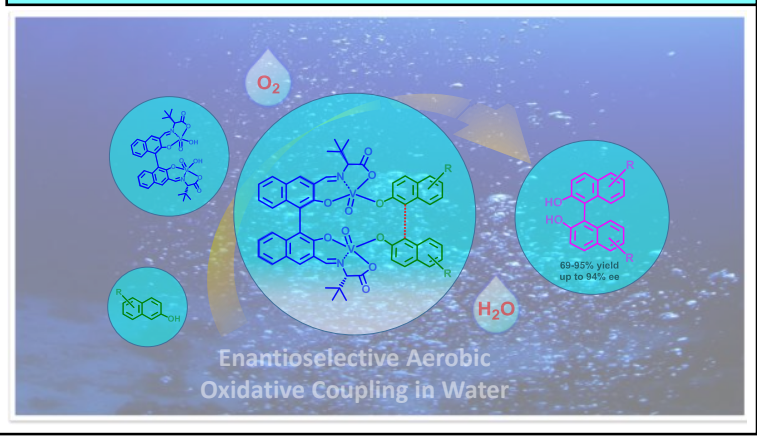

